\title{
Erratum to: Management of duodeno-pancreato-biliary perforations after ERCP: outcomes from an Italian tertiary referral center
}

\author{
Sergio Alfieri - Fausto Rosa $\cdot$ Caterina Cina $\cdot$ Antonio Pio Tortorelli $\cdot$ \\ Andrea Tringali - Vincenzo Perri - Chiara Bellantone · Guido Costamagna • \\ Giovanni Battista Doglietto
}

Published online: 19 April 2013

(C) Springer Science+Business Media New York 2013

\section{Erratum to: Surg Endosc}

DOI 10.1007/s00464-012-2702-9

The name of an author, Chiara Bellantone, from the Digestive Surgery Department, was erroneously omitted.

The correct list of authors is as follows:

Sergio Alfieri, Fausto Rosa, Caterina Cina, Antonio Pio Tortorelli, Andrea Tringali, Vincenzo Perri, Chiara Bellantone, Guido Costamagna, and Giovanni Battista Doglietto.

The online version of the original article can be found under doi:10.1007/s00464-012-2702-9.

S. Alfieri · F. Rosa $(\bowtie) \cdot$ C. Cina $\cdot$ A. P. Tortorelli ·

C. Bellantone - G. B. Doglietto

Digestive Surgery Department, Catholic University,

“A. Gemelli” Hospital, Largo A. Gemelli, 8, 00168 Rome, Italy

e-mail: faust.rosa@tiscali.it

A. Tringali · V. Perri · G. Costamagna

Digestive Endoscopy Department, Catholic University,

“A. Gemelli" Hospital, Rome, Italy 